\title{
Detección de Salmonella sp en Carcasas Porcinas en Camales de Lima, Perú
}

\author{
Detection of Salmonella SP in Pork Carcasses in Slaughterhouses in Lima, Peru \\ Guillermo Salvatierra R. ${ }^{1,2}$, Chris Pinto J. ${ }^{1}$, Edwin Inga E. ${ }^{1}$, Juan Siuce M. ${ }^{1}$, \\ Sonia Calle E. ${ }^{1,3}$
}

\section{Resumen}

El objetivo del presente estudio fue detectar la frecuencia de Salmonella sp, mediante técnicas de aislamiento, en carcasas porcinas destinadas al consumo humano. Se muestrearon 300 carcasas beneficiadas en dos camales de Lima Metropolitana, Perú. Las muestras fueron tomadas mediante hisopados sobre la piel de la cabeza, vientre, lomo y pierna, representando en total 1200 submuestras. Estas fueron transportadas al laboratorio en tubos Falcon con agua peptonada tamponada, donde fueron procesadas siguiendo el protocolo de aislamiento bacteriano basado en la norma ISO 6579:2002. Los aislados fueron identificados mediante pruebas bioquímicas y antisueros específicos. En el $6.3 \pm 2.4 \%$ (19/300) de las carcasas y en 1.8\% (21/1200) de las submuestras se detectó la presencia de Salmonella sp. El mayor porcentaje de aislados se obtuvo de la piel de la cabeza $(33.3 \%, 7 / 21)$ y vientre $(33.3 \%, 7 / 21)$. Los aislados fueron serotipificados e identificados como Salmonella enterica subesp. enterica serotipo Derby. Los resultados confirman la necesidad de implementar medidas de control y detección de la bacteria que permitan reducir la frecuencia de carne de cerdo contaminada que llega al consumidor.

Palabras clave: Salmonella sp, salmonelosis, carcasas porcinas, camales, seguridad alimentaria, salud pública

\section{Abstract}

The aim of this study was to detect the frequency of Salmonella sp by isolation techniques in pork carcasses intended for human consumption. Three hundred carcasses from two slaughterhouses in Lima, Peru were studied. Samples were taken by swabbing the skin of the head, abdomen, back and leg, representing 1200 subsamples. They were

\footnotetext{
${ }^{1}$ Laboratorio de Microbiología y Parasitología Veterinaria, Facultad de Medicina Veterinaria, Universidad Nacional Mayor de San Marcos, Lima, Perú

${ }^{2}$ E-mail: gsalvatierrar@gmail.com

${ }^{3}$ E-mail: calleson@gmail.com
}

Recibido: 30 de setiembre de 2014

Aceptado para publicación: 28 de junio de 2015 
taken to the laboratory in Falcon tubes with buffered peptone water, and processed following the protocol for isolation of bacteria based on ISO 6579:2002. The isolates were identified by biochemical tests and specific antisera. In $6.3 \pm 2.4 \%(19 / 300)$ of carcasses and $1.8 \%(21 / 1200)$ of subsamples were detected Salmonella sp. The highest frequencies of isolates were obtained from the head $(33.3 \%, 7 / 21)$ and the abdomen $(33.3 \%, 7 / 21)$. The isolates were serotyped and identified as Salmonella enterica subesp. enterica serotype Derby. The results confirm the need to implement control measures and detection of the bacteria to reduce the frequency of contaminated pork that reaches the consumer.

Key words: Salmonella sp, salmonellosis, pig carcasses, slaughterhouses, food security, public health

\section{INTRODUCCIÓN}

Salmonella y Campylobacter son los agentes zoonóticos más frecuentemente aislados en casos de gastroenteritis de origen alimentario, tanto en Europa como en los Estados Unidos de Norteamérica (EFSA, 2007). Los casos declarados de tóxico-infecciones por Salmonella en Europa son de 73 por cada 100000 habitantes, con importantes variaciones entre países en función del método diagnóstico, registro de información y hábitos culinarios de cada país. Se estima que la incidencia real de salmonelosis en la Unión Europea puede rondar los 450 casos anuales por cada 100000 habitantes, incluyendo tres muertes por cada millón de personas (Berends et al., 1997).

Salmonella sp pertenece a la familia Enterobacteriaceae, y es conocida como un «patógeno universal» por tener un amplio rango de hospedadores (D'Aoust y Maurer, 2007). Los principales reservorios de Salmonella son los animales portadores asintomáticos, y las fuentes de infección más frecuentes son los alimentos. El aumento de la incidencia de Salmonella sp tiene un gran impacto en la salud pública y la salud animal, y se le ha relacionado con un incremento de la diseminación de microorganismos a través de las cadenas productivas animales (Uribe y Suárez, 2006).
En la industria porcina, la contaminación de la carne por Salmonella puede producirse en cualquier etapa de la cadena cárnica; desde las materias primas para la alimentación del animal, la fabricación de los concentrados, la granja y la planta de sacrificio, hasta los centros de elaboración de productos cárnicos. Diversos estudios han demostrado la contaminación de carcasas porcinas destinadas al consumo humano con Salmonella sp (Käsbohrer et al., 2000; Kranker et al., 2003). Se ha demostrado que cepas de Salmonella llegan a contaminar la superficie de la carcasa durante el proceso de beneficio, existiendo una correlación positiva entre los niveles de Salmonella en las granjas y su presencia sobre la superficie de las carcasas (Käsbohrer et al., 2000). Los errores cometidos en la cadena alimentaria transforman el riesgo potencial en una verdadera multiplicación bacteriana. Debido a eso, el control de este microorganismo en la carcasa es importante para evitar la diseminación del agente a los seres humanos (Lindner, 1995).

El aislamiento microbiológico de Salmonella sp mediante la utilización de medios de cultivo y la posterior identificación bioquímica han sido los métodos más usados para el diagnóstico. De forma general, el método microbiológico utilizado con mayor frecuencia, por su alta sensibilidad, se basa en la norma ISO 6579 (2012). Asimismo, algunas cepas de Salmonella sp se pueden confundir con otras enterobacterias produc- 
toras de $\mathrm{SH}_{2}$ como Proteus mirabilis y Citrobacter freundii, que son comensales del aparato digestivo del hombre y de los animales de sangre caliente, pero que no son enteropatógenos (Terragno et al., 2003). La identificación final de las serovariedades es el resultado de la combinación de las características bioquímicas y de los antígenos somáticos $\mathrm{O}$ y flagelares $\mathrm{H}$ determinados por serología (Grimont y Weill, 2007).

El control de Salmonella es primordial, debido a su importancia como problema de salud pública y a la existencia de barreras comerciales de índole sanitarias en la importación de carne. Diversos estudios epidemiológicos han confirmado la importancia de la carne de cerdo como vector de Salmonella, aunque sin llegar a desplazar a los productos aviares como los principales implicados en estas toxiinfecciones (Hald et al., 2004).

El objetivo del presente estudio fue determinar la presencia de especies de Salmonella, mediante técnicas de aislamiento, en carcasas porcinas destinadas al consumo humano.

\section{Materiales y Métodos}

Las muestras fueron tomadas de 300 carcasas porcinas en dos camales ubicados en el Cercado de Lima y en el distrito de Chorrillos, ambos en la región de Lima, Perú. Los animales procedían de seis granjas ubicadas en Lurín, Puente Piedra, Huaral, Aucallama (2) y Chilca, departamento de Lima. Las muestras fueron procesadas en el Laboratorio de Bacteriología de la Facultad de Medicina Veterinaria (FMV) de la Universidad Nacional Mayor de San Marcos (UNMSM), Lima, Perú.

Los muestreos se iniciaron en marzo de 2012, recolectando muestras semanales de
20 carcasas seleccionadas al azar (280 en el primer camal y 20 en el segundo camal). De cada animal se tomaron cuatro muestras por carcasa (cabeza, lomo, vientre y pierna, respectivamente), según el protocolo descrito por Dorsa et al. (1996), dando un total de 1200 submuestras. El tamaño de muestra se determinó mediante el uso de la fórmula de «Detección de enfermedad» (Mateu y Casal, 2003), considerando un nivel de detección del $1 \%$ de carcasas contaminadas a un nivel de confianza del 95\%, donde el tamaño de la población está indicado por el total de cerdos beneficiados anualmente en Lima, que es 849554 (OEEE, 2012).

Las muestras fueron tomadas cuando las carcasas estaban en la fase de oreo. Se utilizaron hisopos estériles con el método «no destructivo de arrastre». Los hisopos se humedecieron durante cinco segundos antes de la toma de muestra en una solución estéril de agua peptonada tamponada (APT). Luego, con el apoyo de una plantilla de acrílico estéril de $10 \times 10 \mathrm{~cm}$, se tomaron las muestras superficiales en $100 \mathrm{~cm}^{2}$ de cada sección de las carcasas. Se aplicó la mayor presión posible con los hisopos, frotando 10 veces verticalmente y 10 veces horizontalmente cada área delimitada.

Las muestras tomadas se recogieron en tubos falcon con $10 \mathrm{ml}$ de APT y se homogenizaron mediante agitación. Luego, fueron colocadas en una nevera de tecnopor con geles refrigerantes para su transporte a la FMV-UNMSM. Las muestras fueron procesadas siguiendo el protocolo de aislamiento basado en la norma ISO 6579:2002. Los aislados fueron identificados mediantes pruebas bioquímicas y serotipificados con pruebas de aglutinación somática y flagelar. La serotipificación se llevó a cabo en el Laboratorio de Enteropatógenos del Instituto Nacional de Salud (INS) del Perú. Se utilizaron antisueros polivalentes y monovalentes (Denka Seiken, Japón). 


\section{Resultados y Discusión}

En el $6.3 \% \pm 2.4(19 / 300)$ de las carcasas porcinas analizadas se detectó la presencia de Salmonella sp. Asimismo, de las 1200 submuestras analizadas, se obtuvieron 21 aislados compatibles con Salmonella $\mathrm{sp}$, lo que representa un $1.8 \%$ de positividad del total de submuestras procesadas.

Este porcentaje constituye un riesgo de infección en los procesos alimenticios derivados de la carne porcina y tiene gran importancia económica, ya que en el comercio internacional existen normativas que prohíben la presencia de Salmonella en alimentos destinados al consumo humano. Estas indican que cepas de Salmonella deben estar ausentes en $25 \mathrm{~g}$ de carne (ISO, 2012).

En Dinamarca se reporta un 3.2\% (14/ 438) de prevalencia en carcasas porcinas (Arguello et al., 2013), en tanto que en Etiopía se ha reportado una frecuencia de $4 \%$ en carcasas porcinas (Aragaw et al., 2007). Por otro lado, Swanenburg et al. (2001) hallaron una prevalencia de $10 \%$ en carcasas en plantas de beneficio de Holanda. Asimismo, valores más altos han sido reportados en otros países; así, Hotes et al. (2010) reportan $15.7 \%$ en jugos cárnicos de carcasas porcinas en Alemania y Arcos et al. (2013) encontraron una prevalencia de $27.2 \%$ de Salmonella sp en carcasas en plantas de beneficio de Bogotá, Colombia.

Todos los aislados, independientemente de su procedencia, tuvieron la siguiente fórmula antigénica (según el esquema de Kauffman-White): 1,4,[5],12;f,g;[1,2] que corresponde a Salmonella enterica subesp. enterica serotipo Derby (Grimont y Weill, 2007). Esto concuerda con estudios realizados en Dinamarca, que demuestran que los principales aislamientos a partir de carcasas son Salmonella Typhimurium y Salmonella Derby (Sørensen et al., 2004); asimismo, Bahnson et al. (2006) reportan los serotipos Derby (23.2\%) y Typhimurium (12.1\%) como los más frecuentes en mataderos de Midwest, EEUU, y Rostagno et al. (2003) afirman que estos serotipos son frecuentemente aislados en la mayoría de estudios desarrollados en camales.

Por otro lado, Fablet et al. (2006) encontraron una mayor frecuencia de Salmonella Derby seguida por $S$. Typhimurium en granjas en Francia de Salmonella, y Davies et al. (1999) encontraron que Salmonella Derby es el serotipo más común en muestras de heces de porcinos (20/34) en Carolina del Norte, EEUU, lo que podría apoyar la posibilidad de que la contaminación de las carcasas sea a través del contenido intestinal debido a un mal eviscerado.

En relación a la zona corporal de muestreo, el mayor porcentaje de aislamientos se presentó en las muestras de la cabeza $(33.3 \%, 7 / 21)$ y vientre $(33.3 \%, 7 / 21)$, seguidos por el lomo $(23.8 \%, 5 / 21)$ y la pierna $(9.5 \%, 2 / 21)$. La mayor cantidad de aislamientos en la cabeza podría atribuirse a la disposición vertical de la carcasa, con la cabeza hacia el suelo, permitiendo que por gravedad todos los fluidos como agua o sangre discurran por ella; asimismo, se pudo observar durante los muestreos que la cabeza llegaba ocasionalmente a tener contacto con el suelo. Por otro lado, el vientre es una zona primaria de contacto con las vísceras del animal, donde un mal eviscerado podría ocasionar la contaminación de esa zona.

Todas las carcasas contaminadas con Salmonella sp (19/300) procedieron del primer camal. La contaminación microbiana puede darse durante el transporte del animal de la granja a la planta de beneficio, periodo de espera y en cualquiera de las etapas del beneficio, incluyendo el escaldado, depilado, eviscerado, duchado, oreo y almacenamiento en frío (Borch et al., 2002). Diversos estudios sugieren que la contaminación cruzada durante el sacrificio de los animales es una fuente importante de contaminación con Salmonella para las carcasas de animales 
de lotes seronegativos (Swanenburg et al., 2001) y que la alta prevalencia de Salmonella sp en las superficies de las carcasas es indicativo de defectos en el equipo o higiene pobre en la línea de beneficio (Käsbohrer et al., 2000). Por otra parte, Berends et al. (1997) describen una asociación estadística nula entre la prevalencia de Salmonella en nódulos linfáticos mesentéricos (seropositivos) y la contaminación de la carcasa. De la misma manera, Letellier et al. (2009) demostraron que en el $43 \%$ de los casos (56/129), la serología fue negativa a Salmonella mientras las carcasas fueron positivas, lo cual sugiere una contaminación reciente de los animales durante el transporte o contaminación cruzada de las carcasas en las plantas de sacrificio.

En el primer camal no se observó una buena desinfección ni una rotación adecuada de las herramientas entre lotes de animales durante el proceso de eviscerado. Asimismo, fue común observar que las carcasas eran manipuladas de manera excesiva y sin uso de guantes, cuando eran trasladadas a lo largo de los rieles a las zonas de oreo. Otros factores de riesgo importante que fueron observados, y que puede asociarse con la presencia de Salmonella, fue la deficiente higiene del personal del camal y en la limpieza de los pisos, el flujo y densidad de animales, la presencia de insectos y aves silvestres, el mal manejo de la temperatura. El segundo camal, que sí cumplía con las reglas sanitarias establecidas, mostró limpieza y buenas normas de trabajo para el procesamiento de las carcasas. Estas comparaciones, pese al limitado número de carcasas evaluadas, podrían explicar la ausencia de aislamientos positivos en el segundo camal.

Es importante concientizar al personal manipulador de carne porcina de emplear las buenas prácticas de manufactura. Se sabe que el número de Salmonella sp en la superficie de las carcasas podría reducirse con procedimientos cuidadosos en el sacrificio, como el escaldado individual y el retiro cuidadoso del tracto gastrointestinal (Nowak $e t$ al., 2007).
Se ha demostrado que Salmonella Typhimurium y Salmonella Derby son los serotipos más comunes en productos porcinos reconocidos como fuente de transmisión de la salmonelosis humana (Berends et al., 1997; Gebreyes et al., 2004). A su vez, en España, se ha relacionado el aislamiento de cepas de Salmonella Derby de muestras clínicas de origen humano con el consumo de productos derivados del ganado porcino, lo que demuestra la importancia de este serotipo en la salud pública (Valdezate et al., 2005).

\section{Conclusiones}

- Existe evidencia de contaminación por Salmonella sp de las carcasas porcinas destinadas al consumo humano procedentes de camales de Lima metropolitana.

- El 6.3\% (19/300) de las carcasas porcinas estuvieron contaminadas con Salmonella enterica subespecie enterica serotipo Derby.

\section{Literatura Citada}

1. Aragaw K, Molla B, Muckle A, Cole L, Wilkie E, Poppe C, Kleer J, et al. 2007. The characterization of Salmonella serovars isolated from apparently healthy slaughtered pigs at Addis Ababa abattoir, Ethiopia. Prev Vet Med 82: 252-261. doi: 10.1016/ j.prevetmed.2007.05.022

2. Arcos E, Mora L, Fandiño L, Rondón I. 2013. Prevalencia de Salmonella spp en carne porcina, plantas de beneficio y expendios del Tolima. Orinoquia [online] 17(1). [Internet]. Disponible en: http:// www.scielo.org.co/scielo.php?script= sci_art text\&pi d = S 0121 37092013000100007\&lng=es

3. Arguello H, Sørensen G, Carvajal A, Baggesen DL, Rubio P, Pedersen K. 2013. Prevalence, serotypes and resistance patterns of Salmonella in 
Danish pig production. Res Vet Sci 95: 334-342. doi: 10.1016/j.rvsc.2013.04.001

4. Bahnson P, Damman D, Isaacson R, Miller G, Weigel R, Troutt F. 2006. Prevalence of serovars of Salmonella enterica isolated from ileolic lymph nodes of market pigs reared in selected Midwest US swine herds. J Swine Health Prod 14: 182-188.

5. Berends BR, Van Knapen F, Snijders JM, Mossel DA. 1997. Identification and quantification of risk factors regarding Salmonella spp on pork carcasses. Int J Food Microbiol 36: 199-206. doi: 10.1016/ S0168-1605(97)01267-1

6. Borch E, Nesbakken T, Christensen H. 2002. Hazard identification in swine slaughter with respect to foodborne bacteria. Int J Food Microbiol 30: 9-25. doi: 10.1016/0168-1605(96)00988-9

7. D'Aoust JY, Maurer J. 2007. Salmonella species. In: Doyle MP, Beuchet LR (eds). Food microbiology. Fundamentals and frontiers. $3^{\text {rd }} \mathrm{ed}$. Washington DC, USA: ASM Press. p 187-236.

8. Davies RH, Mclaren IM, Bedford S. 1999. Observations on the distribution of Salmonella in pig abattoirs. Vet Rec 145: 655-661. doi: 10.1136/vr.145.23.655

9. Dorsa WJ, Cutter CN, Siragusa GR. 1996. Evaluation of six sampling methods for recovery of bacteria from beef carcass surfaces. Lett Appl Microbiol 22: 39-41. doi: 10.1111/j.1472765X.1996.tb01104.x

10. [EFSA] European Food Safety Authority. 2007. The Community Summary Report on trends and sources of zoonoses, zoonotic agents, antimicrobial resistance and foodborne outbreaks in the European Union in 2006. EFSA 12(2): 3547. doi: 10.2903/j.efsa. 2007.130r

11. Fablet $C$, Robinault $C$, Eono F, Dorenlor V, Labbé A, Fravalo $P$, Madec F. 2006. Factors associated with Salmonella contamination of finishing facilities following cleaning and disinfection procedure. Proc XIX International Pig
Veterinary Society (IPVS) Congress. Copenhagen, Denmark.

12. Gebreyes WA, Davies PR, Turkson PK, Morrow WE, Funk JA, Altier C. 2004. Salmonella enterica serovars from pigs on farms and after slaughter and validity of using bacteriologic data to define herd Salmonella status. J Food Prot 67: 691-697.

13. Grimont PA, Weill FX. 2007. Antigenic formulas of the Salmonella serovars. $9^{\text {th }}$ ed. WHO Collaborating Centre for Reference and Research on Salmonella Instituto Pasteur. [Internet]. Disponible en: http://www.pasteur.fr/ip/portal/action/ WebdriveActionEvent/oid/01s-000036-089

14. Hald T, Vose D, Wegener HC, Koupeev T. 2004. A Bayesian approach to quantify the contribution of animalfood sources to human salmonellosis. Risk Anal 24: 255-269.

15. Hotes S, Kemper N, Traulsen I, Rave G, Krieter J. 2010. Risk factors for Salmonella infection in fattening pigs An evaluation of blood and meat juices samples. Zoonoses Publ Hlth 57: 30-38. doi: 10.1111/j.1863-2378.2010.01361.x

16. ISO. 2012. ISO 6579:2002. Microbiology of food and animal feeding stuffs. Horizontal method for the detection of Salmonella spp [Internet]. Disponible en: http://www.iso.org/iso/ catalogue_detail.htm?csnumber $=29315$

17. Käsbohrer A, Protz D, Helmuth R, Nöckler K, Blaha T, Conraths FJ, Geue L. 2000. Salmonella in slaughter pigs of German origin: an epidemiological study. Eur J Epidemiol 16: 141-146.

18. Kranker S, Alban L, Boes J, Dahl J. 2003. Longitudinal study of Salmonella enterica serotype Typhimurium infection in three Danish farrow-to-finish swine herds. J Clin Microbiol 41: 2282-2288. doi: 10.1128/JCM.41.6.2282-2288.2003

19. Letellier A, Beauchamp G, Guévremont S, D'allaire S, Hurnik D, Quessy S. 2009. Risk factors at slaughter associated with presence of Salmonella on hog carcasses in Canada. J Food Prot 72: 2326-2331. 
20. Lindner E. 1995. Toxicología de los alimentos. $2^{\mathrm{a}}$ ed. Zaragoza: Acribia. $262 \mathrm{p}$.

21. Mateu E, Casal J. 2003. Tamaño de la muestra. Rev Epidem Med Prev 1: 8-14.

22. Nowak B, von Müffling T, Chaunchom S, Hartung J. 2007. Salmonella contamination in pigs at slaughter and on the farm: a field study using an antibody ELISA test and a PCR technique. Int J Food Microbiol 115: 259267. doi: 10.1016/j.ijfoodmicro. 2006. 10.045

23. [OEEE] Oficina de Estudios Económicos y Estadísticos. 2012. Perú: Beneficio de ganado porcino en centros de beneficio según región, 2011-2012. En: Producción Pecuaria e Industria Avícola. Lima: Ministerio de Agricultura y Riego. Perú. p 66.

24. Rostagno MH, Hurd HS, McKean JD, Ziemer CJ, Gailey JK, Leite RC. 2003. Preslaughter holding environment in pork plants is highly contaminated with Salmonella enterica. Appl Environ Microbiol 69: 4489-4494. doi: 10.1128/ AEM.69.8.4489-4494.2003

25. Sørensen L, Alban L, Nielsen B, J. Dahl. 2004. The correlation between
Salmonella serology and isolation of Salmonella in Danish pigs at slaughterhouses. Vet Microbiol 101: 131141. doi: 10.1016/j.vetmic.2004.02.016

26. Swanenburg M, Urlings HAP, Snijders JMA, Keuzenkamp DA, van Knapen F. 2001. Salmonella in slaughter pigs: prevalence, serotypes and critical control points during slaughter in two slaughterhouses. Int J Food Microbiol 70: 243-254. doi: 10.1016/S0168-1605(01) 00545-1

27. Terragno $R$, Caffer M, Bruno $S$, Binsztein N. 2003. Salmonella: aislamiento, identificación y serotipificación. En: Manual de procedimientos. Argentina: Instituto Nacional de Enfermedades Infecciosas - ANLIS «Dr. Carlos G. Malbrán». $46 \mathrm{p}$.

28. Uribe C, Suárez MC. 2006. Salmonelosis no tifoidea y su transmisión a través de alimentos de origen aviar. Colomb Med 37(2): 151-158.

29. Valdezate S, Vidal A, Herrera-León S, Pozo J, Rubio P, Usera MA, et al. 2005. Salmonella Derby clonal spread form pork. Emerg Infect Dis 11: 694698. doi: 10.3201/eid1105.041042 